\title{
How to Estimate the Efficacy of Periodic Control of an Infectious Plant Disease
}

\author{
HORST R. THIEME* \\ Sonderforschungsbereich 123, \\ Universität Heidelberg, Im Neuenheimer Feld 294, \\ D-6900 Heidelberg, Bundesrepublik Deutschland \\ AND \\ J. A. P. HEESTERBEEK \\ Centre for Mathematics and Computer Science \\ P.O. Box 4079, 1009 AB Amsterdam, The Netherlands
}

Received 8 February 1988; revised 25 June 1988

\begin{abstract}
Certain infectious plant diseases are controlled by inspection and subsequent hand removal of diseased parts. In this paper we give two sets of criteria from which one can conclude whether this control effort is adequate or not. These criteria do not require knowledge of the infection- or detection rate of the disease but only use the structure of the contact matrix. Computer experiments give a feeling of how many inspections are needed in order to draw a conclusion.
\end{abstract}

\section{INTRODUCTION}

A widely accepted control measure of an infectious plant disease is sanitation by inspection and roguing [1]. This treatment is applied foremost to diseases with relatively limited dispersion rate (over larger distances) and to diseases against which no good curative chemicals are available. The control consists of inspecting the host plants on a regular basis and removing diseased individuals (or their diseased parts). Examples are control of citrus tristeza virus of citrus trees, the bacterial disease "fire blight" (Erwinia amylovora) in pear and apple orchards, and the fungal disease "silver leaf" (Stereum purpureum) in plum.

Other applications of this control measure are in post entry quarantine inspections [2] and in controlling epidemics of dangerous diseases in an area with many fields of host plants, by protection services. In the former case imported plant material is grown in a greenhouse and sanitated by inspec-

*Supported by a Heisenberg scholarship of the Deutsche Forschungsgemeinschaft. 
tion and roguing until one is certain that the disease, if present, has been eradicated. In the latter case entire fields or greenhouse crops can be "removed" from the host area if infection is present. Examples are inspections for the narcissus nematode in narcissus fields and chrysanthemum white rust (Puccina horiana) in greenhouses.

As only a part of the infected host plants (or fields) is actually detected at a certain inspection and the detection rate is unknown, there is need for criteria from which one can conclude whether the control effort is adequate or not. One would like to know either way after as few inspections as possible. In this paper we will give two sets of these criteria.

\section{THE MODEL}

Fishman and Marcus [3] present a model for an infectious plant disease spreading in an orchard of citrus trees planted in rows. The state of the orchard is inspected periodically, and detected infected plants are removed. Considering each row of trees as a homogeneous unit, the model relates the number of eliminations in every row at the present inspection to those of the previous inspection. Instead of rows of trees one could also think of fields in a certain area as homogeneous units.

The analytical and numerical studies of Fishman and Marcus [3] show that one cannot judge the efficacy of the control by just looking at the elimination numbers from some selected rows. Whether or not the control is adequate, the number of detected infected trees can increase in some rows and decrease in others. We will show that one should combine data from all rows in a specific way and compare these combinations from period to period. A great advantage is that the way of combining the data only depends on the structure of the model and is largely independent of the precise values of the parameters.

For completeness we briefly describe the model of Fishman and Marcus. We restrict our discussion from the very beginning to the situation where the level of infection is still low. This allows us to assume that the fraction of susceptibles is approximately 1 in every row and to write down a linear model.

Let $x_{j}(t)$ denote the fraction of infected ( $=$ infective) trees at time $t$ in row $j$. The spread of the disease is then described by

$$
\frac{d}{d t} x_{j}=\alpha \sum_{k=1}^{N} x_{k} f(|j-k|) .
$$

Here $\alpha>0$ is the relative rate of infection, $N$ is the number of rows, and $f(|j-k|)$ gives the rate at which infective inoculum is transported from row $k$ to row $j$. Note that we assume both isotropy and homogeneity, i.e., the 
transport rate depends only on the distance between the rows. In particular there is no preference of direction in which the disease spreads (due to wind, for example). For generalizations relaxing this assumption, see Section 4.

Periodically the trees are tested for the presence of the disease, and detected infected trees are eliminated. After scaling time we may assume there is one time unit between inspections, i.e., (1.1) holds in the time intervals $[m, m+1), m=0,1, \ldots$. At any time $m \geqslant 1$ we reset the initial conditions with

$$
x_{j}\left(m^{+}\right)=(1-\beta) \cdot x_{j}\left(m^{-}\right) .
$$

Here $m^{-}\left(m^{+}\right)$indicates the limit from the left (right), and $0<\beta<1$ indicates the probability of detection of an infected tree.

The fraction of trees that are detected (and eliminated) in row $j$ during the $m$ th inspection is given by

$$
y_{j}(m):=\beta \cdot x_{j}\left(m^{-}\right) \quad m \geqslant 1, j=1, \ldots, N .
$$

Note that $y_{j}(m)$ is what one actually observes in the orchard.

We combine the distance function $f$ into a matrix $F$,

$$
F=(f(|j-k|))_{1 \leqslant j, k \leqslant N}
$$

and define the vectors

$$
x(t)=\left(x_{1}(t), \ldots, x_{N}(t)\right)^{T}, \quad y(m)=\left(y_{1}(m), \ldots, y_{N}(m)\right)^{T} .
$$

Then (1.1), (1.2), (1.3) take the form

$$
\begin{aligned}
\frac{d}{d t} x & =\alpha F x \quad \text { on }[m, m+1) \\
x\left(m^{+}\right) & =(1-\beta) \cdot x\left(m^{-}\right) \\
y(m) & =\beta \cdot x\left(m^{-}\right) .
\end{aligned}
$$

Solving (1.6) on $[m, m+1)$ we find

$$
x\left(m^{-}\right)=e^{\alpha F} x\left((m-1)^{+}\right)
$$

hence

$$
y(m)=(1-\beta) e^{\alpha F} \cdot y(m-1) .
$$

In the next section we study the behavior of solutions of (1.10). 


\section{QUALITATIVE BEHAVIOR}

We impose a rather natural condition on the contact matrix $F$. We assume $F$ is positive.

ASSUMPTION 2.0

$$
f(0)>0, f(1)>0, \text { and } f(j) \geqslant 0, \quad j=2, \ldots, N-1 .
$$

This implies that a power $F^{p}$ of $F$ is strictly positive, i.e., all entries of $F^{p}$ are larger than zero. Let $r(F)$ denote the spectral radius of $F$,

$$
r(F)=\inf _{n \rightarrow \infty}\left\|F^{n}\right\|^{1 / n}
$$

Note that $r(F)>0$.

It is well known that $r(F)$ equals the maximum modulus of the eigenvalues of $F$ and so is independent of the choice of $\|\cdot\|$ in (2.1). Moreover, if we define

$$
G:=(1-\beta) e^{\alpha F}
$$

then assumption 2.0 implies that $G$ is strictly positive and that

$$
r(G)=(1-\beta) e^{\alpha r(F)} .
$$

The next proposition, due to Perron and Frobenius, is important for studying the asymptotic behavior of solutions to (1.10).

PROPOSITION 2.1

(i) $r(F)$ from (2.1) is the only eigenvalue of $F$ with maximum modulus.

(ii) There exists a unique $v \in \mathbb{R}_{\gg 0}^{N}$ such that $F v=r(F) v,\|v\|=1$.

(iii) $r(G)$ from (2.3) is the only eigenvalue of $G$ with maximum modulus.

(iv) $v$ from (ii) is the only vector in $\mathbb{R}_{>0}^{N}$ satisfying $\|v\|=1$ and $G v=r(G) v$.

Here $\mathbb{R}_{\gg 0}^{N}$ and $\mathbb{R}_{>0}^{N}$ denote the sets of strictly positive and positive vectors, respectively. The cone of non-negative vectors will be symbolized by $\mathbb{R}_{\geqslant 0}^{N}$.

For a proof of this, see Bellman [4], chapter 16, theorem 1.2.

We can rewrite (1.10) in terms of $G$,

$$
y(m)=G y(m-1), \quad m \geqslant 2 .
$$

A standard spectral decomposition argument now provides information about the asymptotic behavior of $y(m)$; see Bellman [4], chapter 16, theorem 4. 
Note that, due to the symmetry of $F$ and $G$, the left eigenvector of $G$ equals the right eigenvector.

THEOREM 2.2

Let $v$ be the eigenvector of $F$ and $G$ from Proposition 2.1(ii) and let $y(m)$, $m=2,3, \ldots$, be a solution of $(2.4)$; then

$$
\lim _{m \rightarrow \infty}\left[r(G)^{-m} \cdot y(m)\right]=\langle y(0), v\rangle \cdot v
$$

and further

$$
\langle y(m), v\rangle=r(G) \cdot\langle y(m-1), v\rangle=r(G)^{m} \cdot\langle y(0), v\rangle .
$$

Here $\langle\cdot, \cdot\rangle$ denotes the scalar product in $\mathbb{R}^{N}$.

As $v \in \mathbb{R}_{\gg 0}^{N}$ we have $\langle y(m), v\rangle>0$ for $y(m) \in \mathbb{R}_{>0}^{N}$. So $\langle y(m), v\rangle$ is an admissible measure for the level of "infestation" of the orchard or field. It seems reasonable to call the control successful if the "infestation" decreases in a suitable sense and goes extinct in the long run. This is the content of Corollary 2.3, which follows directly from Theorem 2.2 .

COROLLARY 2.3

(i) If $r(G)<1$ the control succeeds, i.e., $y(m) \rightarrow 0$ for $m \rightarrow \infty$ and $\langle y(m), v\rangle$ strictly decreases to 0 for $m \rightarrow \infty$.

(ii) If $r(G)>1$, the control does not succeed; in particular $\langle y(m), v\rangle$ strictly increases.

Here $v$ is the eigenvector from Proposition 2.1.

So if $\alpha, \beta$, and $F$ are known one can calculate $r(G)$ and check whether the control succeeds or not. Calculating $r(G)$ by a standard computer program is much more efficient than simulating Equation (1.10).

If only $F$ is known, one can calculate $v$ at least numerically and determine $r(G)$ from the data by forming scalar products with $y(m)$ and $y(m-1)$. This requires data from two different inspections, see Equation (2.6).

The most realistic situation, however, is the one in which all three factors are unknown. We will discuss this in Section 3.

\section{CRITERIA AND RULES FOR SUCCESS OR FAILURE OF CONTROL}

We now turn to the situation where $\alpha, \beta$, and $F$ are unknown and we only know the structure of $F$, i.e., $F$ is of the form (1.4) and satisfies assumption 2.0. As $F$ is symmetric so is $G$, and

$$
\|G y\| \leqslant r(G) \cdot\|y\|
$$


for all $y \in \mathbb{R}^{N}$. Here $\|\cdot\|=\langle\cdot, \cdot\rangle^{1 / 2}$ is the Euclidean norm. From (3.1) and Corollary 2.3 we derive the following criterion for failure of control.

THEOREM 3.1

If $\|y(m)\|>\|y(m-1)\|$ for some $m \geqslant 2$, the control fails.

A criterion for success follows from Lemma A.1 in the appendix. THEOREM 3.2

If $y(m)<y(m-1)$ for some $m \geqslant 2$, the control succeeds.

Here $y(m)<y(m-1)$ means $y_{j}(m) \leqslant y_{j}(m-1)$ for all $j=1, \ldots, N$ and $y_{j}(m)<y_{j}(m-1)$ for at least one $j$.

Theorem 2.2 implies that, unless $r(G)=1$, either the criterion in Theorem 3.1 or that in Theorem 3.2 will be met if one waits sufficiently long. There is, however, a great disadvantage. It may take too long for the criteria to show up and the disease may by then have already damaged the whole field severely. So we look for rules that may be a bit less reliable than the criteria in Theorems 3.1 and 3.2 but come into operation much sooner.

To this end we recall equation (2.6), which allows us to determine $r(G)$ from $y(m)$ and $y(m-1)$ provided we know the eigenvector $v$. The basic idea is to replace the now unknown $v$ by two vectors $\underset{\sim}{v}, \tilde{v}$, and to form scalar products with $y(m)$.

We choose $\underset{\sim}{v}$ and $\tilde{v}$ as follows:

$$
\begin{aligned}
& \underset{\sim}{v_{j}}:=\sin \left(\pi \frac{j}{N+1}\right), \quad j=1, \ldots, N, \\
& \tilde{v}_{j}:=\sin \left(\pi \frac{j+n-1}{N+2 n-1}\right), \quad j=1, \ldots, N .
\end{aligned}
$$

Here $n$ is related to $N$ by $N=2 n$ or $N=2 n+1$. The choice of $\underset{\sim}{v}$ is suggested by the fact that $\underline{v}$ is an eigenvector of $G$ when $f(k)=0$ for all $k \geqslant 2$, i.e., if there is nearest-neighbor infection only. See Maruyama [5], section 10.3, and Othmer and Scriven [6], table 1. Note that $\underset{\sim}{v}$ and $\tilde{v}$ do not depend on parameters of the model.

By combining Corollary A.3 and Proposition A.5 from the appendix we have

THEOREM 3.3

Let $N \geqslant 4$ and let $u(m)$ be a solution to (2.4), i.e., $u(m)=G \cdot u(m-1)$, $m=2,3, \ldots$. Then

$$
\frac{\langle u(m), \tilde{v}\rangle}{\langle u(m-1), \tilde{v}\rangle} e^{-\alpha \epsilon_{N}} \leqslant r(G) \leqslant \frac{\langle u(m), \underset{\sim}{v}\rangle}{\langle u(m-1), \underset{\sim}{v}\rangle} e^{\alpha \epsilon_{N}}
$$


for all $m=2,3, \ldots$ with

$$
\epsilon_{N}=\frac{2 \pi^{2}}{(N+1)^{2}} \sum_{k=1}^{N-1} k^{2} \cdot f(k) .
$$

It is reasonable to assume that $\sum_{k=1}^{\infty} k^{2} f(k)<\infty$ because the contribution of infectious inoculum from row $j$ to row $l$ will decrease practically exponentially with the distance between $j$ and $l$. Then $\epsilon_{N} \rightarrow 0$ as (constant)/ $(N+1)^{2}$ and $\exp \left(\alpha \epsilon_{N}\right) \rightarrow 1$ for $N \rightarrow \infty$ quite fast.

So it seems justified to formulate the following rule.

RULE 3.4

Let $N$ be large

(i) If $\langle y(m), \underset{\sim}{v}\rangle\langle\langle y(m-1), \underset{\sim}{v}\rangle$, for one $m \geqslant 2$, the control succeeds.

(ii) If $\langle y(m), \tilde{v}\rangle\rangle\langle y(m-1), \tilde{v}\rangle$ for one $m \geqslant 2$, the control fails.

Actually, via the formula in Theorem 3.3, the data from any two subsequent inspections provide estimates of the critical value $r(G)$. By Theorem 2.2 both

$$
\frac{\langle u(m), \tilde{v}\rangle}{\langle u(m-1), \tilde{v}\rangle} \quad \text { and } \quad \frac{\langle u(m), \underset{\sim}{v}\rangle}{\langle u(m-1), \underset{\sim}{v}\rangle}
$$

converge toward $r(G)$.

Though this convergence may be different for different initial infections, there is an order of convergence which is independent of the initial infection because $\langle u(m), \tilde{v}\rangle=\left\langle u(1), G^{m-1} \tilde{v}\right\rangle$ and the same relation holds for $\underset{\sim}{v}$.

The same remark applies to Rule 3.4. Again Theorem 2.2 guarantees that either Rule 3.4(i) or 3.4(ii) will come into operation sooner or later unless $r(G)=1$. How long it will take will crucially depend on where the initial infection occurs. Nevertheless there is a number $m$ for which the rules work independently of the initial infection, namely, the first $m$ such that $G^{m} \underline{v}<$ $G^{m-1} \underset{\sim}{v}$ or $G^{m} \tilde{v}>G^{m-1} \tilde{v}$.

As we are not able to find a satisfactory analytic estimate of how fast either Rule 3.4(i) or (ii) will work, we perform a computer experiment. We solve Equation (2.4) numerically. From the scalar products of $y(m)$ with $\underset{\sim}{v}$ and $\tilde{v}$ we find $m$ such that Rule 3.4(i) or (ii) applies. We also look for $m$ such that one of the criteria in Theorem 3.1 or Theorem 3.2 applies. All parameters but $\beta$ are chosen as in [3], i.e., $F=(f(|j-k|))$ with $f(k)=$ $\exp (-k / \gamma), \gamma=0.8, \alpha=0.58, N=30$; and $\beta$ is varied. Numerically calculating $r(G)$ one finds $r(G)=1$ if $\beta \approx 0.778=: \beta^{*}$. 
TABLE 1

The Control Succeeds, $\beta>\beta^{*}$

\begin{tabular}{|c|c|c|c|c|c|c|c|c|c|c|c|c|}
\hline$\beta$ & & & & 0.78 & & & & & & & & \\
\hline$j$ & 1 & 2 & 3 & 4 & 5 & 6 & 7 & 8 & 9 & & & \\
\hline$m$ & 9 & 8 & 7 & 6 & 2 & 2 & & & & & & \\
\hline$\beta$ & & & 0.785 & & & & & & & & 0.79 & \\
\hline$j$ & 1 & 2 & 3 & 4 & 5 & 6 & & & & 1 & 2 & \\
\hline$m$ & 4 & 4 & 2 & 2 & & & & & & 3 & 2 & 2 \\
\hline$\beta$ & & & 0.795 & & & & & & & 0.8 & & \\
\hline$j$ & 1 & 2 & 3 & & & & & & & & 2 & \\
\hline$m$ & 3 & 2 & 2 & & & & & & & 2 & 2 & \\
\hline
\end{tabular}

TABLE 2

The Control Fails, $\beta<\beta^{*}$

\begin{tabular}{lrrrrrrrrrr}
\hline$\beta$ & \multicolumn{10}{c}{0.775} \\
\cline { 2 - 8 } & 1 & 2 & 3 & 4 & 5 & 6 & 7 & 8 & 9 & 10 \\
$m_{r}$ & 14 & 14 & 13 & 12 & 11 & 8 & 2 & 2 & & \\
$m_{c}$ & $\geqslant 20$ & $\geqslant 20$ & $\geqslant 20$ & $\geqslant 20$ & $\geqslant 20$ & 20 & 18 & 16 & &
\end{tabular}

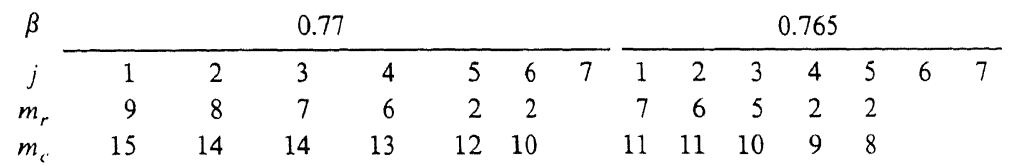

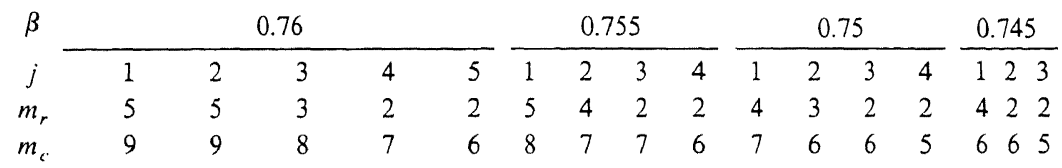

\begin{tabular}{|c|c|c|c|c|c|c|c|c|c|c|c|c|c|c|c|}
\hline$\beta$ & \multicolumn{3}{|c|}{0.74} & \multicolumn{3}{|c|}{0.735} & \multicolumn{3}{|c|}{0.73} & \multicolumn{3}{|c|}{0.725} & \multicolumn{3}{|c|}{0.72} \\
\hline f & 1 & 2 & 3 & 1 & 2 & 3 & 1 & 2 & 3 & 1 & 2 & 3 & 1 & 2 & 3 \\
\hline$m_{r}$ & 3 & 2 & 2 & 3 & 2 & 2 & 3 & 2 & 2 & 3 & 2 & 2 & 2 & 2 & 2 \\
\hline$m_{c}$ & 6 & 5 & 4 & 5 & 5 & 4 & 5 & 4 & 4 & 5 & 4 & 3 & 5 & 4 & 3 \\
\hline
\end{tabular}


As a superposition principle holds due to linearity of the model, it is only necessary to look at the situation where the disease is initially present in a single row. The special form of matrix $F$ implies that the spread is completely symmetric whether the disease starts in row $j$ or in row $N+1-j$.

The numerical experiments confirm that Rule 3.4 is reliable, i.e., $\langle u(m), v\rangle$ increases in $m$ if the control fails $[r(G)>1]$ and $\langle u(m), \tilde{v}\rangle$ decreases in $m$ if the control succeeds $[r(G)<1]$.

As for the number of inspections it takes before the rules apply, we list our results in Tables 1 and 2 . In Table 1 we look at cases in which the control succeeds, i.e., $\beta>\beta^{*}$. The probability of detection is $\beta, j$ is the row of first manifestation of the disease and $m$ the first control period in which Rule 3.4(i) indicates success. In this experiment the criterion in Theorem 3.2 never came into operation before $m=20$. Table 2 illustrates the case in which the control fails, i.e., $\beta<\beta^{*}$. Here $m_{r}$ gives the first period of inspection in which Rule 3.4(ii) works, and $m_{c}$ the first period where the criterion in Theorem 3.1 works.

\section{DISCUSSION}

In Section 3 we established criteria (Theorems 3.1 and 3.2) and rules (Rule 3.4) for judging the efficacy of an epidemic control (a "rule" may not be completely reliable, in contrast to a "criterion"). We have shown analytically that Rules 3.4(i) and 3.4(ii) are close to reliability if the number of rows is large enough. The reliability of Rule 3.4 has been confirmed by numerical experiments for an example from the literature, i.e., citrus tristeza virus in a citrus orchard. The rules in 3.4 are more efficient than the criteria of Theorems 3.1 and 3.2. In view of the fact that our experiment concerned a case where the epidemic increases or decreases slowly, Rule 3.4 gives quite fast information about whether the control succeeds or fails. In particular, Rule 3.4(i) works well. It may happen that the control succeeds although Rule 3.4(i) does not yet apply, but this indicates that the disease decreases very slowly and that the control effort should be increased in order to eradicate the disease in a reasonable time.

From Tables 1 and 2 we see that both the rules and the criteria are at their worst if the initial infection is close to or at the outer rows.

As is always the case with models, we have worked in an ideal world. We assumed that once the epidemic has started there is no further infection from outside the field. If the host is grown in a greenhouse this assumption can be justified, but if we have an outdoor field of hosts it is realistic only in cases where infectious inoculum is transported badly over large distances. However, in cases where inoculum spreads rapidly and easily over larger distances, for example, windborne inoculum, one is not likely to apply control by inspection and roguing. Exceptions are cases where few better alternatives are available. 
The model also excludes any bias in the direction of spread (by wind, for example). Here a modification of our approach seems possible; see Maruyama [5], section 10.3, (10.41). However, this requires knowledge of the bias, and we would lose the nice property of Rule 3.4 that it does not require any knowledge of the parameters.

Furthermore the model considers any row as a homogeneous unit and so makes the problem one-dimensional in space. In certain cases a generalization to a more complicated geometric situation may be possible. See Othmer and Scriven [6].

An assumption that is not always realistic is that the entire infected individual is removed. In many cases if infection is not too severe, only the infected branch of the tree is removed, for example, in the case of fire blight in pear. If we assume that the branch is removed in a proper way (i.e., not leaving an infected piece still attached to the tree), we can still apply our model. In other cases, however, not only the entire individual but also, as a precaution, the trees in a certain area around this tree are removed. This can be the case with faster spreading diseases that one wants to eradicate completely.

Furthermore in an actual orchard the fruit grower will frequently plant rows of different cultivars, differing among other things in vulnerability to the disease. In inspecting highly vulnerable rows the grower will be more attentive to disease symptoms. This causes $\beta$ to be dependent on the row. Also in a real-life situation $\alpha$ will vary with the weather.

Not only our model reflects an ideal world; this also holds for our experiments. In a real-world situation one faces stochastic effects, errors in data collection, variation in the control effort, etc. Rule 3.4 must still be tested in a real-world situation by comparison with actual field data or with a pseudostochastic computer simulation. Actual field data to compare with our Rule 3.4 were not yet available for this paper.

\section{APPENDIX}

Let $A$ be a strictly positive $N \times N$ matrix, i.e., if $A=\left(a_{i j}\right)$ then $a_{i j}>0$ $\forall i, j=1=1, \ldots, N$. For convenience we introduce the following notation. Let $x, y \in \mathbb{R}^{N}$; then

$$
\begin{aligned}
& x \geqslant y \text { iff } x_{j} \geqslant y_{j}, \quad \forall j=1, \ldots, N \\
& x>y \text { iff } x \geqslant y, \quad x \neq y \\
& x \gg y \text { iff } x_{j}>y_{j}, \quad \forall j=1, \ldots, N .
\end{aligned}
$$

The following way of estimating $r(A)$, the spectral radius of $A$, is well known. We give a short proof for completeness. 
LEMMA A.I

(i) Let $A x>\lambda x, \lambda \in \mathbb{R}, x>0$ then $r(A)>\lambda$.

(ii) Let $A x<\lambda x, \lambda \in \mathbb{R}, x>0$ then $r(A)<\lambda$.

Proof. We only prove (i); the proof of (ii) is similar. Let $y=A x$. As $A$ is strictly positive we have $y \gg 0$ and $A y \gg \lambda y$ so $\exists \epsilon>0: A y \gg(\lambda+\epsilon) y$, but then we have $r(A) \geqslant \lambda+\epsilon$ (by chapter 16, theorem 2 of [4]), and the result follows.

We now assume that $A$ is a symmetric matrix. The following easy theorem is the key for estimating $r(A)$ from the data.

THEOREM A.2

Let $\lambda_{1} x \leqslant A x$ and $\lambda_{2} y \geqslant A y ; \lambda_{1}, \lambda_{2} \geqslant 0$ and $x, y \gg 0$. Then

$$
\begin{gathered}
\lambda_{1} \leqslant r(A) \leqslant \lambda_{2} \\
\frac{\langle A u, x\rangle}{\langle u, x\rangle} \geqslant \lambda_{1} \geqslant r(A)+\lambda_{1}-\lambda_{2}, \forall u>0 \\
\frac{\langle A u, y\rangle}{\langle u, y\rangle} \leqslant \lambda_{2} \leqslant r(A)+\lambda_{2}-\lambda_{1}, \forall u>0 .
\end{gathered}
$$

Proof. The first statement follows directly from Bellman ([4], chapter 16, theorem 2). For the second statement we use the symmetry of $A$ :

$$
\forall u \geqslant 0: \quad\langle A u, x\rangle=\langle u, A x\rangle \geqslant\left\langle u, \lambda_{1} x\right\rangle=\lambda_{1}\langle u, x\rangle
$$

so

$$
\frac{\langle A u, x\rangle}{\langle u, x\rangle} \geqslant \lambda_{1} \geqslant \lambda_{1}+r(A)-\lambda_{2}, \quad \forall u>0
$$

as $r(A)-\lambda_{2} \leqslant 0$ by (i). Statement (iii) is proved analogously.

We now look at the matrix $G=(1-\beta) e^{\alpha F}$ with $F$ satisfying Assumption 2.0. Note that $G$ is symmetric and strictly positive.

COROLLARYA.3

Let $\mu_{1} x \leqslant F x$ and $\mu_{2} y \geqslant F y$ with $\mu_{1}, \mu_{2} \in \mathbb{R}$ and $x, y \gg 0$, then

$$
\begin{gathered}
(1-\beta) e^{\alpha \mu_{1}} \leqslant r(G) \leqslant(1-\beta) e^{\alpha \mu_{2}} \\
\frac{\langle G u, y\rangle}{\langle u, y\rangle} e^{\alpha\left(\mu_{1}-\mu_{2}\right)} \leqslant r(G) \leqslant \frac{\langle G u, x\rangle}{\langle u, x\rangle} e^{\alpha\left(\mu_{2}-\mu_{1}\right)}
\end{gathered}
$$


Proof. Obviously $G x \geqslant(1-\beta) e^{\alpha \mu_{1}} \cdot x$ and $G y \leqslant(1-\beta) e^{\alpha \mu_{2}} \cdot y$. The first statement then follows from Theorem A.2. By Theorem A.2(ii) we have

$$
\begin{aligned}
\frac{\langle G u, x\rangle}{\langle u, x\rangle} & \geqslant r(G)+(1-\beta)\left(e^{\alpha \mu_{1}}-e^{\alpha \mu_{2}}\right) \\
& =r(G)+(1-\beta) e^{\alpha \mu_{2}}\left(e^{\alpha\left(\mu_{1}-\mu_{2}\right)}-1\right) \\
& \geqslant r(G) e^{\alpha\left(\mu_{1}-\mu_{2}\right)} .
\end{aligned}
$$

The second part of inequality (ii) follows analogously from Theorem A.2(iii).

In view of this corollary we look for $x, y \gg 0$ and $\mu_{1}, \mu_{2} \in \mathbb{R}$ such that $\mu_{1} x \leqslant F x$ and $\mu_{2} y \geqslant F y$ and $\left|\mu_{1}-\mu_{2}\right|$ as small as possible. We choose $\underset{\sim}{v}$ for $x$ and $\tilde{v}$ for $y$ with

$$
\begin{aligned}
& \underline{\sim}_{j}:=\sin \left(\pi \frac{j}{N+1}\right), \quad j=1, \ldots, N \\
& \text { and } \quad \tilde{v}_{j}:=\sin \left(\pi \frac{j+n-1}{N+2 n-1}\right), \quad j=1, \ldots, N .
\end{aligned}
$$

As $\sin x=\sin (\pi-x)$ for $0 \leqslant x \leqslant \pi, \underset{\sim}{v}$ has the symmetry $\underset{\sim}{v}=\underset{\sim}{v_{N+1-j}}$, $j=1, \ldots, N$. It is easy to see that $F$ preserves this symmetry, i.e., $\left(F_{v_{j}}\right)=$ $(F \underline{\sim})_{N+1-j}$.

We can now prove the following technical proposition.

PROPOSITION A.4

(i) For $\underset{\sim}{v_{j}}=\sin [\pi j /(N+1)], j=1, \ldots, N$, we have $\mu_{1} \underset{\sim}{v} \leqslant F_{\sim}^{\underset{\sim}{v} \text { with }}$

$$
\mu_{1}=f(0)+2 \sum_{k=1}^{n} f(k) \cos \left(\frac{\pi k}{N+1}\right)
$$

and $N=2 n$ or $N=2 n+1$.

(ii) For $\tilde{v}_{j}=\sin [\pi(j+n-1) /(N+2 n-1)], j=1, \ldots, N$, we have $\mu_{2} \tilde{v} \geqslant$ Fũ with

$$
\mu_{2}=f(0)+2\left[\sum_{k=1}^{n-1} f(k) \cos \left(\frac{\pi k}{N+2 n-1}\right)+\sum_{k=n}^{N-1} f(k) \sin \left(\frac{\pi k}{N+2 n-1}\right)\right]
$$

and $N=2 n$ or $N=2 n+1$.

Proof.

(i) By the fact that $F$ preserves the symmetry $\underset{\sim}{\underset{v}{v}}={\underset{\sim}{v+1-j}}_{N+j}, j=1, \ldots, N$, we only need consider $\mu_{1} \underset{\sim}{v} \leqslant F \underline{v}$ for the index $j=1, \ldots, n$ (if $N=2 n$ ) or 
$j=1, \ldots, n+1$ (if $N=2 n+1$ ). Suppose $j$ satisfies this restriction. Then

$$
\begin{aligned}
(F \underset{\sim}{v})_{j} & =\sum_{k=1}^{N} f(|k-j|) \sin \left(\frac{\pi k}{N+1}\right) \\
& =\sum_{l=1-j}^{N-j} f(|l|) \sin \left(\frac{\pi(l+j)}{N+1}\right) \geqslant \sum_{l=1-j}^{n} f(|l|) \sin \left(\frac{\pi(l+j)}{N+1}\right) .
\end{aligned}
$$

It now follows that

$$
(F \underset{\sim}{v})_{j} \geqslant \sum_{l=-n}^{n} f(|l|) \sin \left(\frac{\pi(l+j)}{N+1}\right)
$$

because $i-j \geqslant-n$ and $\sin [\pi(l+j) /(N+1)] \leqslant 0$ for $l=-n, \ldots,-j$.

By the addition rule for the sine function we get from $(\dagger)$

$$
\begin{aligned}
\left(F_{\sim}^{v}\right) j \geqslant & \cos \left(\frac{\pi j}{N+1}\right) \sum_{l=-n}^{n} f(|l|) \sin \left(\frac{\pi l}{N+1}\right) \\
& +\sin \left(\frac{\pi j}{N+1}\right) \sum_{l=-n}^{n} f(|l|) \cos \left(\frac{\pi l}{N+1}\right) \\
={\underset{\sim}{j}}_{j} & \cdot\left[f(0)+\sum_{l=1}^{n} f(l) \cos \left(\frac{\pi l}{N+1}\right)\right] .
\end{aligned}
$$

This proves statement (i).

(ii) The second statement is proved in an analogous way. We then find $\mu_{2}^{*} \tilde{v} \geqslant F \tilde{v}$ with

$$
\begin{aligned}
\mu_{2}^{*}= & f(0)+2 \sum_{k=1}^{n-1} f(k) \cos \left(\frac{\pi k}{N+2 n-1}\right) \\
& +\sum_{k=n}^{N-1} f(k)\left[\cos \left(\frac{\pi k}{N+2 n-1}\right)+\sin \left(\frac{\pi k}{N+2 n-1}\right)\right]
\end{aligned}
$$

for $k \geqslant n$ we can write

$\cos \left(\frac{\pi k}{N+2 n-1}\right) \leqslant \cos \left(\frac{\pi n}{N+2 n-1}\right) \leqslant \cos \left(\frac{\pi}{4}\right)=\sin \left(\frac{\pi}{4}\right) \leqslant \sin \left(\frac{\pi k}{N+2 n-1}\right)$.

Substituting this in the expression for $\mu_{2}^{*}$, we get $\mu_{2}$. Then $\mu_{2} \geqslant \mu_{2}^{*}$, so $\mu_{2} \tilde{v} \geqslant F \tilde{v}$ holds and the statement is proved. 
Note that $\mu_{2}^{*}$ in the proof of Proposition A.4(ii) is a better estimate than $\mu_{2}$. However, we look for an estimation of $\mu_{2}-\mu_{1}$ which gives a good feeling for how $\mu_{2}-\mu_{1}$ decrease as the number of rows $N$ tends to infinity.

PROPOSITION A.5

Let $N \geqslant 4$, then

$$
\mu_{2}-\mu_{1} \leqslant \frac{2 \pi^{2}}{(N+1)^{2}} \sum_{k=1}^{N-1} k^{2} \cdot f(k)
$$

Proof. From Proposition A.4 we have

$$
\begin{aligned}
\mu_{2}-\mu_{1} \leqslant & 2 \sum_{k=1}^{n-1} f(k)\left[\cos \left(\frac{\pi k}{N+2 n-1}\right)-\cos \left(\frac{\pi k}{N+1}\right)\right] \\
& +2 \sum_{k=n}^{N-1} f(k) \sin \left(\frac{\pi k}{N+2 n-1}\right) .
\end{aligned}
$$

As $|\cos y-\cos x| \leqslant|x-y| \sin x \leqslant|x-y| x$ for $0 \leqslant y \leqslant x \leqslant \pi / 4$ by the mean value theorem, we obtain

$$
\left|\cos \left(\frac{\pi k}{N+2 n-1}\right)-\cos \left(\frac{\pi k}{N+1}\right)\right| \leqslant \pi^{2} \frac{k^{2}}{(N+1)^{2}} .
$$

Furthermore, for $k \geqslant n$,

$$
\sin \left(\frac{\pi k}{N+2 n-1}\right) \leqslant \frac{\pi^{2} k^{2}}{\pi n(N+2 n-1)} \leqslant \frac{\pi^{2} k^{2}}{3 n(N+2 n-1)} \leqslant \frac{\pi^{2} k^{2}}{(N+1)^{2}}
$$

because for $N \geqslant 4$ we have $n \geqslant 2$ (remember, $N=2 n$ or $N=2 n+1$ ) and $3 n \geqslant 2 n+2 \geqslant N+1, N+2 n-1 \geqslant N+1$. Substituting the above estimates in $(\dagger \dagger)$ gives the desired result.

This work was started as part of a student's seminar run by K. Schumacher and one of the authors in Heidelberg in winter 1985-86. The computer program used in the numerical experiments at the end of Section 3 was designed in this seminar by Cordula Determann and Stephan Treue. 
Note that $\mu_{2}^{*}$ in the proof of Proposition A.4(ii) is a better estimate than $\mu_{2}$. However, we look for an estimation of $\mu_{2}-\mu_{1}$ which gives a good feeling for how $\mu_{2}-\mu_{1}$ decrease as the number of rows $N$ tends to infinity.

PROPOSITION A.5

Let $N \geqslant 4$, then

$$
\mu_{2}-\mu_{1} \leqslant \frac{2 \pi^{2}}{(N+1)^{2}} \sum_{k=1}^{N-1} k^{2} \cdot f(k) .
$$

Proof. From Proposition A.4 we have

$$
\begin{aligned}
\mu_{2}-\mu_{1} \leqslant & 2 \sum_{k=1}^{n-1} f(k)\left[\cos \left(\frac{\pi k}{N+2 n-1}\right)-\cos \left(\frac{\pi k}{N+1}\right)\right] \\
& +2 \sum_{k=n}^{N-1} f(k) \sin \left(\frac{\pi k}{N+2 n-1}\right) .
\end{aligned}
$$

$s y-\cos x|\leqslant| x-y|\sin x \leqslant| x-y \mid x$ for $0 \leqslant y \leqslant x \leqslant \pi / 4$ by the mean heorem, we obtain

$$
\left|\cos \left(\frac{\pi k}{N+2 n-1}\right)-\cos \left(\frac{\pi k}{N+1}\right)\right| \leqslant \pi^{2} \frac{k^{2}}{(N+1)^{2}} .
$$

aermore, for $k \geqslant n$,

$$
\sin \left(\frac{\pi k}{N+2 n-1}\right) \leqslant \frac{\pi^{2} k^{2}}{\pi n(N+2 n-1)} \leqslant \frac{\pi^{2} k^{2}}{3 n(N+2 n-1)} \leqslant \frac{\pi^{2} k^{2}}{(N+1)^{2}}
$$

ise for $N \geqslant 4$ we have $n \geqslant 2$ (remember, $N=2 n$ or $N=2 n+1$ ) and ! $n+2 \geqslant N+1, N+2 n-1 \geqslant N+1$. Substituting the above estimates in ives the desired result.

'ork was started as part of a student's seminar run by $K$. Schumacher $f$ the authors in Heidelberg in winter 1985-86. The computer program e numerical experiments at the end of Section 3 was designed in this y Cordula Determann and Stephan Treue. 


\section{REFERENCES}

1 J. C. Zadoks and R. D. Schein, Epidemiologv and Plant Disease Management, Oxford University Press, 1979.

2 G. H. Berg, Post-entry and intermediate quarantine stations. In: Plant Heath and Quarantine in International Transfer of Genetic Resources, W. B. Hewitt and L. Chiajappa, eds., Boca Raton, Florida, 1977, pp. 315-326.

3 S. Fishman and R. Marcus, A model for spread of plant disease with periodic removals, J. Math. Biol. 21:149-158 (1984).

4 R. Bellman, Introduction to Matrix Analysis, McGraw-Hill, New York, 1960.

5 T. Maruyama, Stochastic Problems in Population Genetics, Lecture Notes in Biomathematics, Vol. 17, Springer-Verlag, New York, 1977.

6 H. G. Othmer and L. G. Scriven, Instability and dynamic pattern in cellular networks, J. Theor. Biol. 32:507-537 (1971). 